\title{
Waist Circumference and Risk of Liver Cancer: A Systematic Review and Meta-Analysis of over 2 Million Cohort Study Participants
}

\author{
Jamal Rahmani ${ }^{a} \quad$ Hamed Kord Varkaneh $^{b}$ Vasileios Kontogiannis ${ }^{c}$ \\ Paul M. Ryan ${ }^{d}$ Hiba Bawadie Somaye Fatahi ${ }^{f}$ Yong Zhang ${ }^{g}$ \\ ${ }^{a}$ Department of Community Nutrition, Student Research Committee, Faculty of Nutrition \\ and Food Technology, National Nutrition and Food Technology Research Institute, Shahid \\ Beheshti University of Medical Sciences, Tehran, Iran; 'b Students' Scientific Research Center \\ (SSRC), Tehran University of Medical Sciences (TUMS), Tehran, Iran, Tehran, Iran; 'Institute \\ of Health \& Society, Newcastle University, Newcastle upon Tyne, UK; ${ }^{d}$ School of Medicine, \\ University College Cork, Cork, Ireland; e Department of Human Nutrition, College of

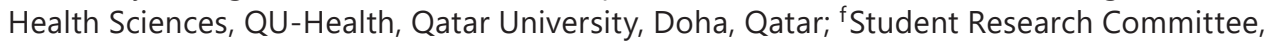 \\ Department of Nutrition, School of Public Health, Iran University of Medical Sciences, \\ Tehran, Iran; ${ }^{9}$ School of Public Health and Health Management, Chongqing Medical \\ University, Chongqing, China
}

\section{Keywords}

Liver cancer · Waist circumference $\cdot$ Central obesity

\begin{abstract}
Purpose: Liver cancer is the sixth most common type of cancer worldwide, and waist circumference (WC) is associated with its risk beyond body mass index (BMI). This dose-response meta-analysis was performed to investigate the association between WC and the risk of incident liver cancer using prospective cohort studies. Methods: A comprehensive systematic search was conducted in MEDLINE/PubMed, Web of Science databases, Scopus, and Cochrane from inception to May 2019. Studies with retrospective or prospective cohort design that reported hazard ratio (HR), risk ratio, or odds ratio, and the corresponding 95\% confidence intervals $(\mathrm{Cl})$ for liver cancer based on WC categories were included in this meta-analysis. Combined HRs with $95 \% \mathrm{Cls}$ was estimated by DerSimonian and Laird random-effects models. Results: Associations between WC and liver cancer were reported in 5 articles with 2,547,188 participants. All studies were published between 2013 and 2019. Pooled results showed a strong significant association with minimum heterogeneity between WC and risk of liver cancer (HR 1.59, 95\% Cl 1.38-1.83, $p_{\text {heterogeneity }}=0.42: I^{2}=0 \%$ ). Moreover, a dose-response model indicated a significant positive association between WC and risk of liver cancer $(\exp (b)=$ $1.018, p<0.001)$. Conclusions: This systematic review and dose-response meta-analysis highlights WC as a significant risk factor related to the incidence of liver cancer.
\end{abstract}




\section{Liver Cancer}

\section{Introduction}

Liver cancer is the sixth most common type of cancer worldwide, with more than 840,000 new cases in 2018 and with men having a higher chance of developing the disease than women [1]. In the last 3 decades, the average age in patients diagnosed with liver cancer has risen, and the proportion of female patients with liver cancer has been slightly increasing [2]. The disease has poor survival rates, and in Europe the mean age-standardized survival rate at 5 years is $12 \%$ [3]. In addition, the fact that the disease does not produce any obvious symptoms, meaning that it is diagnosed at an advanced stage, makes the identification of a biomarker or predictive measure of paramount importance. Approximately $80 \%$ of the cases occur in less developed countries such as Asia and Africa, while Europe and Latin America and the Caribbean report the lowest incidences [1]. This elevated incidence in developing countries can be primarily attributed to the high prevalence of chronic infection with hepatitis B virus [4], which is one of the main risk factors for developing liver cancer. However, another major risk factor of liver cancer is the nonalcoholic fatty liver disease and the more severe nonalcoholic steatohepatitis, both of which are linked to increased generalized and central adiposity $[2,5]$.

Previous studies indicated an increased association between measures of adiposity such as waist circumference (WC), waist-to-hip ratio, and body mass index (BMI) and liver cancer [6-9]. Although BMI is an indicator of adiposity, WC has been shown to be a more accurate predictor of abdominal fat compared to BMI in males [8]. However, although the studies examining the link between this measure of adiposity and liver cancer indicate an increased risk of liver cancer with higher WC, they have been conducted in different regions of the world (China, South Korea, and USA) with different patient characteristics. Furthermore, WC plays an important role in the metabolic syndrome, which is diagnosed by several parameters such as WC and triglycerides, HDL, blood pressure, and fasting glucose levels [10]. WC $>102 \mathrm{~cm}$ in men and $>88 \mathrm{~cm}$ in women are cutoff points for the metabolic syndrome [11]. A previous meta-analysis indicated a relationship between the metabolic syndrome and the risk of liver cancer [12]. Furthermore, studies showed a higher incidence of liver cancer in individuals with a higher WC, and thus a higher WC may predict a higher risk of liver cancer $[13,14]$.

Thus, in the current systematic review and meta-analysis, we initially aimed to review the literature of cohort studies looking at the association between WC and liver cancer and pool together all the evidence to explore whether a higher WC results in a higher risk of liver cancer.

\section{Materials and Methods}

\section{Search Strategy}

The present study was conducted based on the recommendations of the Meta-Analysis of Observational Studies in Epidemiology (MOOSE) guidelines [15]. In May 2019, a comprehensive literature search of relevant studies was done in several databases (PubMed/MEDLINE, Scopus, Cochrane library, and Web of Science databases) without any time and language restriction. The search strategy, which is presented in online supplementary Table 1 (for all online suppl. material, see www.karger.com/doi/10.1159/000502478), was designed to contain all terms relevant to "waist circumference" and "liver cancer." The reference lists of relevant papers were in turn scrutinized to identify further relevant studies.

\section{Inclusion Criteria}

Studies were included in this meta-analysis if they met the following inclusion criteria: (1) retrospective or prospective cohort design, and data on (2) hazard ratio (HR), risk ratio (RR), or odds ratio (OR) as well as the corresponding 95\% confidence interval (CI) for liver cancer based on WC categories. Cross-sectional, case-control, and intervention studies, conference reports, reviews, editorials, nonhuman studies, case reports, and ecological studies were excluded. 


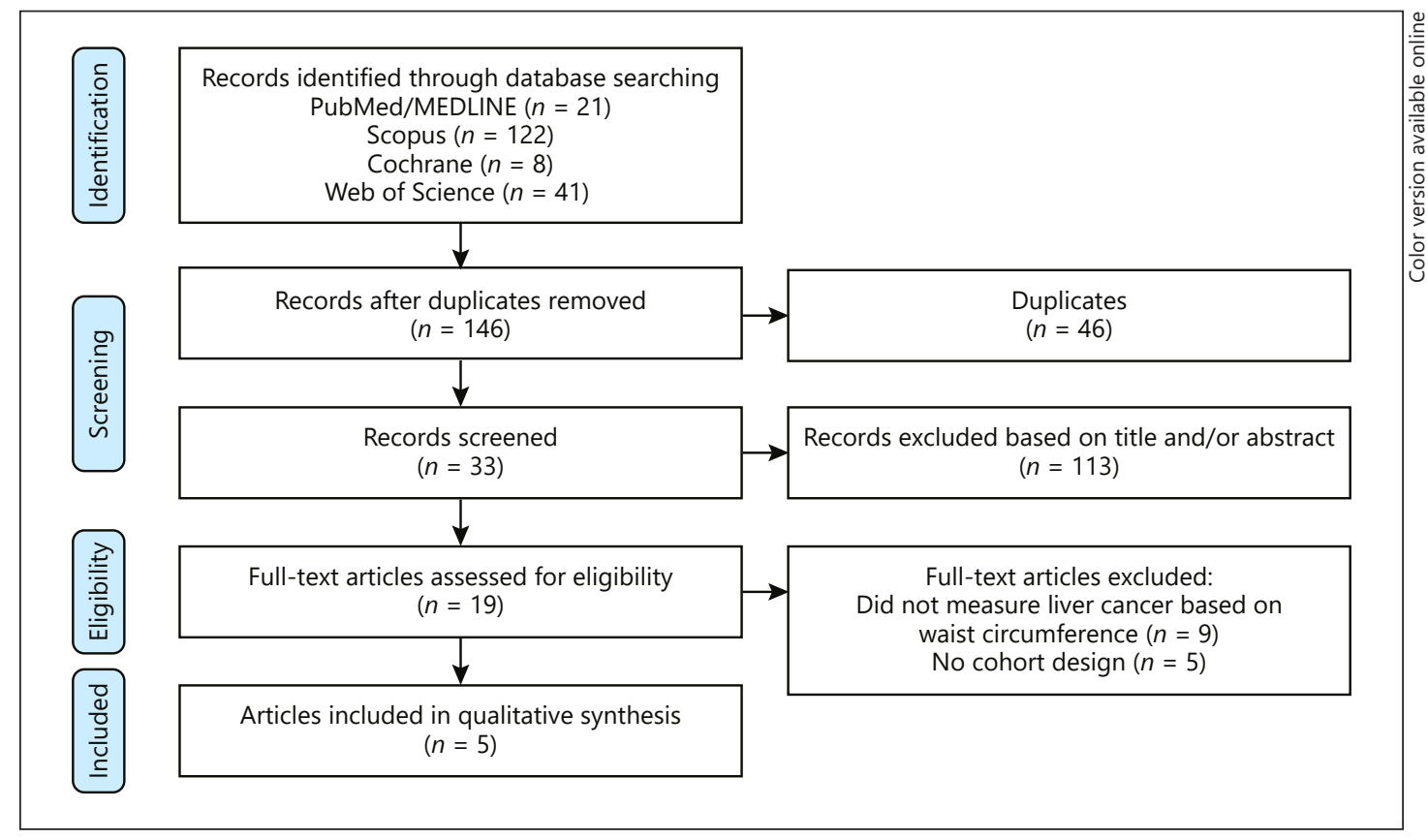

Fig. 1. Flow chart of included studies.

Data Extraction and Quality Assessment

Two authors (J.R. and H.K.V.) screened all studies based on title and abstract independently. Full texts of relevant studies were reviewed and discrepancies between authors were resolved by the senior author (Y.Z.). Extraction forms were used to extract the following data from the studies included: first author, cohort location, year of publication, cohort name, number of participants, age of participants, fully adjusted model of summary estimates, and 95\% CIs of the liver cancer risk. The quality of the studies included was evaluated by the Newcastle-Ottawa Quality Assessment Scale (NOS) [16].

\section{Statistical Analysis}

DerSimonian and Laird random effect models were used to combine the results of the studies [17]. The lowest WC category in each study was considered as the reference category in combination risk estimates. The Cochrane Q test and $I^{2}$ statistics were used to evaluate heterogeneity among the studies included. Restricted cubic splines with 3 knots at the 10th, 50th, and 90th\% percentiles of the distribution were conducted to assess the relationship between predictors and response [18]. Sensitivity analysis was performed to investigate the effect of each study on overall analysis. Funnel plot, Begg's rank correlation test, and Egger's regression asymmetry test were used to detect potential publication bias. All statistical analyses were conducted using Stata 14.0 statistical software (Stata Corporation, College Station, TX, USA), and a value of $p<0.05$ was considered significant.

\section{Results}

\section{Literature Search}

Figure 1 represents the flow diagram of the systematic search undertaken. Following a comprehensive search and removal of duplicate studies, 146 studies were compiled for initial screening. From these, 113 irrelevant records were excluded, and a further 14 studies were excluded based on inclusion and exclusion criteria after evaluation of the remaining full texts. Finally, 5 studies with 2,547,188 participants met the inclusion criteria and were included in this meta-analysis $[8,13,14,19,20]$. 
Rahmani et al.: Waist Circumference and Risk of Liver Cancer

Table 1. Baseline characteristics of the 5 studies included in the meta-analysis

\begin{tabular}{|c|c|c|c|c|c|c|c|}
\hline \multirow{2}{*}{$\begin{array}{l}\text { First } \\
\text { author }\end{array}$} & \multirow[t]{2}{*}{ Year } & \multirow[t]{2}{*}{ Country } & \multirow[t]{2}{*}{ Cohort name } & \multicolumn{3}{|l|}{ Participants } & \multirow{2}{*}{$\begin{array}{l}\text { Follow-up, } \\
\text { years }\end{array}$} \\
\hline & & & & $n$ & $\begin{array}{l}\text { age, } \\
\text { years }\end{array}$ & gender & \\
\hline Pang [13] & 2019 & China & China Kadoorie Biobank & 512,713 & 51 & both & 10.0 \\
\hline Wei [14] & 2018 & China & Kailuan male cohort study & 104,825 & 51.4 & male & 8.9 \\
\hline Lee [19] & 2016 & South Korea & $\begin{array}{l}\text { Historical cohort in Bundang } \\
\text { Jesaeng Hospital }\end{array}$ & 102 & 46.4 & both & 3.8 \\
\hline Campbell [8] & 2016 & US & NCI cohort & $1,570,023$ & 58.2 & both & - \\
\hline Schlesinger [20] & 2013 & $\begin{array}{l}\text { European } \\
\text { countries }\end{array}$ & EPIC study & 359,525 & 52 & both & 8.6 \\
\hline
\end{tabular}

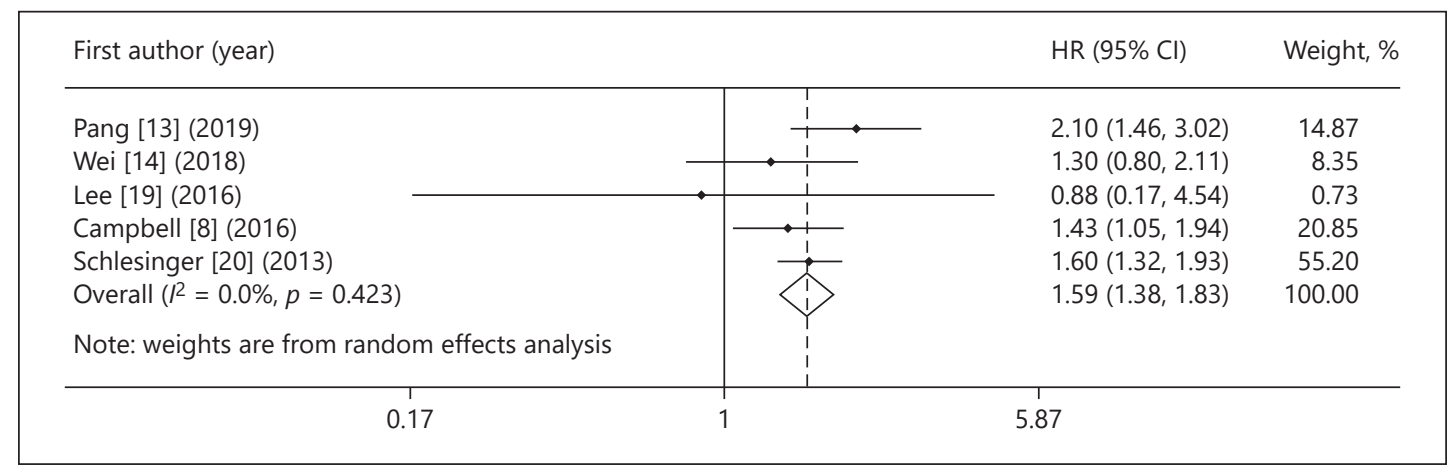

Fig. 2. The forest plot of waist circumference (highest category versus lowest one) and risk of liver cancer.

\section{Study Characteristics and Quality Assessment}

Table 1 outlines general characteristics of the studies included. All studies were published between 2013 and 2019, two studies were conducted in China [13, 14], 1 in South Korea [19], 1 in the USA [8], and 1 in European countries [20]. Participants were aged 46-58 years, with a mean age of 51 years. The mean follow-up of participants in the studies was 7.8 years. One study was conducted solely in a male cohort [14], while the remaining 4 included both genders $[8,13,19,20]$. Online supplementary Table 2 provides information on the quality of the studies, and most studies had high quality with NOS scores. The mean quality of the studies included was 7 (ranging from 5 to 9). Most studies had appropriate follow-up length. In all studies, the groups with and without risk factor exposure were drawn from the same community.

\section{Main Results of the Meta-Analysis}

Five studies with 2,547,188 participants reported an increased risk of liver cancer based on WC $[8,13,14,19,20]$. Combined results of the studies included with random-effects model suggested that the highest WC category was significantly associated with an increased risk of liver cancer (HR 1.59, 95\% CI 1.38-1.83, $p_{\text {heterogeneity }}=0.42: I^{2}=0 \%$ ) compared to the lowest WC category (Fig. 2).

\section{Dose-Response Analysis}

A dose-response analysis was conducted on 3 studies with 942,392 participants $[13,14$, $20]$. The pooled HR from the linear dose-response model indicated a significant positive asso- 


\section{Liver \\ Cancer}

Fig. 3. Linear dose-response relationship between waist circumference and the risk of liver cancer.

Fig. 4. Nonlinear dose-response relationship between waist circumference and the risk of liver cancer.

\begin{tabular}{l}
\hline Liver Cancer 2020;9:6-14 \\
\hline DOI: 10.1159/000502478
\end{tabular}

Rahmani et al.: Waist Circumference and Risk of Liver Cancer
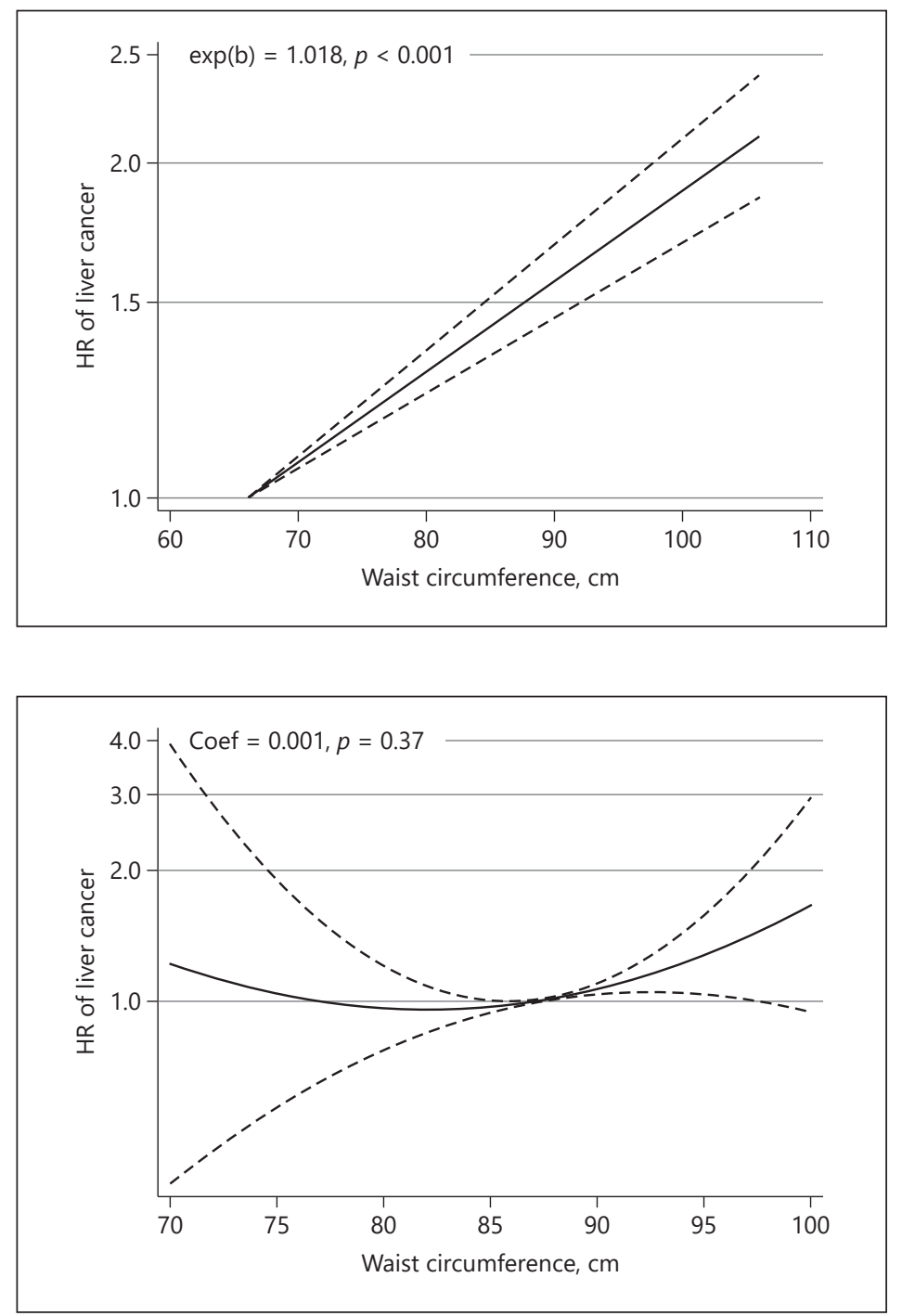

ciation between $\mathrm{WC}$ and the risk of liver cancer $(\exp (\mathrm{b})=1.018, p<0.001)$ (Fig. 3), while a nonlinear dose-response analysis did not identify any significant association between WC and the risk of liver cancer (coefficient of variation $=0.001, p=0.37$ ) (Fig. 4).

\section{Publication Bias and Sensitivity Analysis}

Funnel plots did not show significant asymmetry between the studies included (Fig. 5). Furthermore, Begg's and Egger's regression test did not show any significant publication bias ( $p=0.62$ and $p=0.60$, respectively). Sensitivity analysis did not show significant differences beyond the limits of $95 \%$ CI between calculated HRs for the studies (online suppl. Fig. 1).

\section{Discussion}

In the present study, we aimed to compile and review the available literature from relevant cohort studies assessing whether central adiposity, assessed by WC, is associated with an increased risk of liver cancer. To the best of our knowledge, this study is the first 


\section{Liver Cancer}

Fig. 5. Funnel plots of waist circumference and the risk of liver cancer with pseudo $95 \%$ confidence intervals. Begg's test $=$ 0.62 ; Egger's regression test $=$ 0.60 .

\begin{tabular}{|c|c|}
\hline \multicolumn{2}{|l|}{ Liver Cancer 2020;9:6-14 } \\
\hline DOI: $10.1159 / 000502478$ & $\begin{array}{l}\text { (c) } 2019 \text { The Author(s). Published by S. Karger AG, Basel } \\
\text { www.karger.com/lic }\end{array}$ \\
\hline
\end{tabular}

Rahmani et al.: Waist Circumference and Risk of Liver Cancer

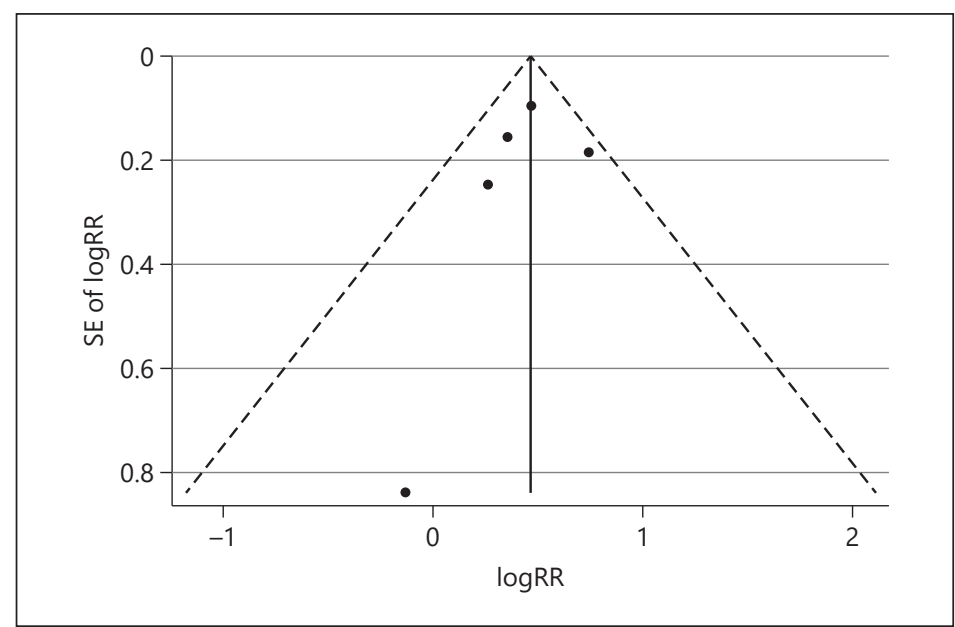

meta-analysis that investigates the relationship between WC and liver cancer; previous studies investigated the relationship between BMI and liver cancer [21,22], but since BMI has several limitations to show adiposity; $W C$ is a better parameter $[23,24]$. The analysis, which included data from 5 cohort studies with 2,547,188 participants, revealed that participants falling within the highest WC category were at significantly higher risk of having a diagnosis of liver cancer than those in the lowest category. Moreover, a subset analysis of nearly 1 million participants uncovered a significant linear dose-response relationship between continuous WC and the risk of liver cancer.

Both in terms of the global scale and the resistance to intervention, obesity and its related sequelae represent one of the greatest public health challenges of the 21st century [25]. In addition to the dysfunctions related to the metabolic syndrome and increased central adiposity, such as insulin resistance, dyslipidemia, and hypertension, the obese phenotype also carries with it an elevated risk of several types of malignancies from breast to colon cancer [26, 27]. In line with this, the burden of liver cancer has also increased concomitant with the rising obesity rates from 1975 to 2012 [28]. As mentioned above, the increasing incidence of liver cancer makes it uniquely fatal in nature, with previous estimates ranking it as the second leading cause of cancer-related death globally [29]. At the center of this relationship lies the pathogenesis of nonalcoholic fatty liver disease and nonalcoholic steatohepatitis, which represent 2 stages of hepatic dysfunction leading to irreversible liver cirrhosis and ultimately hepatocellular carcinoma, which accounts for $85-90 \%$ of primary liver cancers [30]. This process is in part driven by Western-style dietary intake and lifestyle impairments. Therefore, several meta-analyses have demonstrated a clear association between liver cancer and BMI, which follows a nonlinear dose-response [22, 31, 32]. However, only relatively recently, WC, a parameter which represents central or abdominal obesity more closely, has attracted attention as a predictor of liver cancer.

Indeed, central obesity has been found to be a major independent risk factor in the development of a range of cardiometabolic conditions [33]. Although BMI remains the traditional method applied to assess obesity in the clinic, its relevance to obesity-related health risks is highly disputable. In fact, one observational study of nearly 15,000 NHANES III participants found that WC clearly outperformed BMI in predicting hypertension, dyslipidemia, and the metabolic syndrome [23]. Indeed, this characteristic has been confirmed in several other demographic groups, e.g., postmenopausal women [34].

The first of the cohorts assessed within this analysis, the European Prospective Investigation into Cancer and Nutrition study, probed for associations of several anthropometric 
indices of obesity and metabolic dysfunction with the most commonly diagnosed primary liver cancers in 359,525 European participants [20]. The investigators found that waist circumference was associated with hepatocellular carcinoma independently of the diabetes status and heavy alcohol intake. Similarly, a subsequent pooled analysis of a demographically diverse cohort of $>1.5$ million US participants by Campbell et al. [8] revealed that 5 -cm incremental increases in WC were associated with a higher risk of liver cancer (HR 1.08, 95\% CI 1.04-1.13) again independent of the diabetes status. Finally, the most recent analysis of 0.5 million East-Asian participants by Pang et al. [13] confirmed that WC was one of the most significant anthropometric indicators of the liver cancer risk with a HR of 1.13 (95\% CI 1.041.21) per 5 -cm increment and 1.26 (95\% CI 1.09-1.46) per standard deviation from the mean when adjusting for BMI.

The retrospective study conducted by Lee et al. [19] reported on a hepatitis B virus surface antigen (HBsAg)-positive cohort undergoing treatment, making the study population and design somewhat distinct from the other studies included in the meta-analysis. In line with this, the study found no significant association between WC and hepatocellular carcinoma. However, the weight attributed to these data in the present meta-analysis was negligible and, therefore, did not greatly impact the results. Interestingly, within the Kailuan male cohort study, Wei et al. [14] detected that exclusion of HBsAg-positive participants in a subset association analysis corrected the relationship from a U-shape into a linear dose-dependent response. This confounder may explain the failure of both studies to demonstrate a clear relationship between WC and liver cancer in this meta-analysis and also suggests that the metric may only be robust as a screening tool in HBsAg-negative individuals.

Taken together, these results indicate that central obesity is closely associated with the risk of liver cancer and, in turn, may represent a potentially modifiable factor in the primary and secondary prevention of the disease. In line with this, several recommendations may be made to detect associations with early stage liver cancer at both an individual and population scale. Firstly, physicians may wish to introduce regular WC screening into their practice to identify those potentially at risk of liver cancer. In addition, screening tools which incorporate several risk factors associated with primary liver cancer, e.g., WC, could be designed and validated in large populations. Finally, interventions directed towards WC reduction in obese cohorts may be designed in order to assess the putative causal relationship between central obesity and liver cancer.

\section{Strengths and Limitations}

The present study design and execution has several important strengths and limitations. Perhaps the most obvious strength lies in the volume of data assessed within this metaanalysis, with more than 2.5 million participants involved in the 5 studies included. In addition, these studies investigated cohorts with a relatively diverse racial and geographical background, which strengthens the reliability and applicability of the conclusions. Moreover, these studies demonstrate a relatively low degree of heterogeneity, making their inclusion and analysis appropriate. The application of a random-effects model methodology herein adds to the robustness of the result by controlling for several important factors. Lastly, due to the inherent cohort design of the studies included in the present analysis, although we present a clear association, no causation can be directly inferred from these data. Most studies included in this analysis were conducted in both genders and without giving details on the ethnic background. So these parameters were not analyzed in subgroup analyses, which was a limitation of this study. 
Rahmani et al.: Waist Circumference and Risk of Liver Cancer

\section{Conclusion}

In the present review and meta-analysis, we compiled data on WC and liver cancer status from more than 2.5 million individuals from 5 relatively recent studies in order to probe the relationship between central obesity and the malignancy. Herein, we demonstrate a clear positive association between WC and the risk of liver cancer. Moreover, we analyze the linear exposure-dependent nature of this relationship. These data suggest that WC may represent an easily quantified and modifiable risk factor in the development of liver cancer. Future studies should aim to explore a causal relationship as well as opportunities to intervene.

\section{Disclosure Statement}

The authors have no conflict of interest to declare.

\section{Funding Sources}

No funding was received for this study.

\section{Author Contributions}

Jamal Rahmani, Hamed Kord Varkaneh, and Yong Zhang designed the study. The literature search, and data screening and extraction were done by Jamal Rahmani and Hamed Kord Varkaneh. Quality assessment was carried out by Hiba Bawadi and Somaye Fatahi. Jamal Rahmani analyzed and interpreted data. Jamal Rahmani, Yong Zhang, Hiba Bawadi, Vasileios Kontogiannisc, Somaye Fatahi, and Paul M. Ryan wrote and edited the manuscript. All authors read and approved the final manuscript.

\section{References}

1 Liver cancer: How diet, nutrition and physical activity affect liver cancer risk. 2018 [updated 2018 Apr 24]. Available from: https://www.wcrf.org/dietandcancer/liver-cancer.

2 Osaki Y, Nishikawa H. Treatment for hepatocellular carcinoma in Japan over the last three decades: our experience and published work review. Hepatol Res. 2015 Jan;45(1):59-74.

3 De Angelis R, Sant M, Coleman MP, Francisci S, Baili P, Pierannunzio D, et al.; EUROCARE-5 Working Group. Cancer survival in Europe 1999-2007 by country and age: results of EUROCARE-5-a population-based study. Lancet Oncol. 2014 Jan;15(1):23-34.

4 Center M, Siegel R, Jemal A. American Cancer Society Global Cancer: Facts \& Figures. Atlanta (GA): American Cancer Society; 2011.

5 White DL, Kanwal F, El-Serag HB. Association between nonalcoholic fatty liver disease and risk for hepatocellular cancer, based on systematic review. Clin Gastroenterol Hepatol. 2012 Dec;10(12):1342-1359.e2.

6 Diet, nutrition, physical activity and cancer: A global perspective: The third expert report. 2018 [updated 2018 Apr 23]. Available from: https://www.wcrf.org/dietandcancer.

7 Schlesinger S, Aleksandrova K, Pischon T, Fedirko V, Jenab M, Trepo E, et al. Abdominal obesity, weight gain during adulthood and risk of liver and biliary tract cancer in a European cohort. Int J Cancer. 2013 Feb;132(3): 645-57.

8 Campbell PT, Newton CC, Freedman ND, Koshiol J, Alavanja MC, Beane Freeman LE, et al. Body mass index, waist circumference, diabetes, and risk of liver cancer for U.S. adults. Cancer Res. 2016 Oct; 76(20):6076-83.

9 Andreasson A, Carlsson AC, Önnerhag K, et al. Waist/hip ratio better predicts development of severe liver disease within 20 years than body mass index: a population-based cohort study. Clin Gastroenterol Hepatol. 2017;15(8):1294-1301. e2.

10 Després JP, Lemieux I. Abdominal obesity and metabolic syndrome. Nature. 2006 Dec;444(7121):881-7.

11 Arnlöv J, Ingelsson E, Sundström J, Lind L. Impact of body mass index and the metabolic syndrome on the risk of cardiovascular disease and death in middle-aged men. Circulation. 2010 Jan;121(2):230-6.

12 Esposito K, Chiodini P, Colao A, Lenzi A, Giugliano D. Metabolic syndrome and risk of cancer: a systematic review and meta-analysis. Diabetes Care. 2012 Nov;35(11):2402-11. 
13 Pang Y, Kartsonaki C, Guo Y, Chen Y, Yang L, Bian Z, et al. Central adiposity in relation to risk of liver cancer in Chinese adults: A prospective study of 0.5 million people. Int J Cancer. 2019 Sep;145(5):1245-53.

14 Wei L, Li N, Wang G, Feng X, Lyu Z, Li X, et al. Waist Circumference Might Be a Predictor of Primary Liver Cancer: A Population-Based Cohort Study. Front Oncol. 2018 Dec;8:607.

15 Stroup DF, Berlin JA, Morton SC, Olkin I, Williamson GD, Rennie D, et al. Meta-analysis of observational studies in epidemiology: a proposal for reporting. Meta-analysis Of Observational Studies in Epidemiology (MOOSE) group. JAMA. 2000 Apr;283(15):2008-12.

16 Stang A. Critical evaluation of the Newcastle-Ottawa scale for the assessment of the quality of nonrandomized studies in meta-analyses. Eur J Epidemiol. 2010 Sep;25(9):603-5.

17 Jackson D, White IR, Thompson SG. Extending DerSimonian and Laird's methodology to perform multivariate random effects meta-analyses. Stat Med. 2010 May;29(12):1282-97.

18 Harrell FE Jr, Lee KL, Pollock BG. Regression models in clinical studies: determining relationships between predictors and response. J Natl Cancer Inst. 1988 Oct;80(15):1198-202.

19 Lee J, Yoo SH, Sohn W, Kim HW, Choi YS, Won JH, et al. Obesity and hepatocellular carcinoma in patients receiving entecavir for chronic hepatitis B. Clin Mol Hepatol. 2016 Sep;22(3):339-49.

20 Schlesinger S, Aleksandrova K, Pischon T, Fedirko V, Jenab M, Trepo E, et al. Abdominal obesity, weight gain during adulthood and risk of liver and biliary tract cancer in a European cohort. Int J Cancer. 2013 Feb; 132(3): 645-57.

21 Larsson SC, Wolk A. Overweight, obesity and risk of liver cancer: a meta-analysis of cohort studies. Br J Cancer. 2007 Oct;97(7):1005-8.

22 Chen Y, Wang X, Wang J, Yan Z, Luo J. Excess body weight and the risk of primary liver cancer: an updated meta-analysis of prospective studies. Eur J Cancer. 2012 Sep;48(14):2137-45.

23 Janssen I, Katzmarzyk PT, Ross R. Waist circumference and not body mass index explains obesity-related health risk. Am J Clin Nutr. 2004 Mar;79(3):379-84.

24 Janssen I, Katzmarzyk PT, Ross R. Body mass index, waist circumference, and health risk: evidence in support of current National Institutes of Health guidelines. Arch Intern Med. 2002 Oct;162(18):2074-9.

25 Abarca-Gómez L, Abdeen ZA, Hamid ZA, Abu-Rmeileh NM, Acosta-Cazares B, Acuin C, et al.; NCD Risk Factor Collaboration (NCD-RisC). Worldwide trends in body-mass index, underweight, overweight, and obesity from 1975 to 2016: a pooled analysis of 2416 population-based measurement studies in 128.9 million children, adolescents, and adults. Lancet. 2017 Dec;390(10113):2627-42.

26 Morimoto LM, White E, Chen Z, Chlebowski RT, Hays J, Kuller L, et al. Obesity, body size, and risk of postmenopausal breast cancer: the Women's Health Initiative (United States). Cancer Causes Control. 2002 Oct;13(8): 741-51.

27 Larsson SC, Wolk A. Obesity and colon and rectal cancer risk: a meta-analysis of prospective studies. Am J Clin Nutr. 2007 Sep;86(3):556-65.

28 Ryerson AB, Eheman CR, Altekruse SF, Ward JW, Jemal A, Sherman RL, et al. Annual Report to the Nation on the Status of Cancer, 1975-2012, featuring the increasing incidence of liver cancer. Cancer. 2016 May;122(9): 1312-37.

29 Jemal A, Bray F, Center MM, Ferlay J, Ward E, Forman D. Global cancer statistics. CA Cancer J Clin. 2011 Mar-Apr;61(2):69-90.

30 El-Serag HB, Rudolph KL. Hepatocellular carcinoma: epidemiology and molecular carcinogenesis. Gastroenterology. 2007 Jun;132(7):2557-76.

31 Rui R, Lou J, Zou L, Zhong R, Wang J, Xia D, et al. Excess body mass index and risk of liver cancer: a nonlinear dose-response meta-analysis of prospective studies. PLoS One. 2012;7(9):e44522-44522.

32 Wang Y, Wang B, Shen F, Fan J, Cao H. Body mass index and risk of primary liver cancer: a meta-analysis of prospective studies. Oncologist. 2012;17(11):1461-8.

33 Corrigan FE 3rd, Kelli HM, Dhindsa DS, Heinl RE, Al Mheid I, Hammadah M, et al. Changes in truncal obesity and fat distribution predict arterial health. J Clin Lipidol. 2017 Nov - Dec;11(6):1354-1360. e3.

34 Van Pelt RE, Evans EM, Schechtman KB, Ehsani AA, Kohrt WM. Waist circumference vs body mass index for prediction of disease risk in postmenopausal women. Int J Obes Relat Metab Disord. 2001 Aug;25(8):1183-8. 